\title{
Theoretical Calculation of the Low-Lying Electronic States of the Molecule ScSe
}

\author{
Rawad Halabi, Mahmoud Korek ${ }^{*}$ \\ Faculty of Science, Beirut Arab University, Beirut, Lebanon \\ Email: rawadhalabi555@hotmail.com, "fkorek@yahoo.com
}

Received 11 October 2015; accepted 28 December 2015; published 31 December 2015

Copyright (C) 2015 by authors and Scientific Research Publishing Inc.

This work is licensed under the Creative Commons Attribution International License (CC BY). http://creativecommons.org/licenses/by/4.0/

(c) () Open Access

\begin{abstract}
Theoretical investigation of the lowest electronic states of ScSe molecule, in the representation ${ }^{2 s+1} \Lambda^{(+/-)}$, has been performed via CASSCF and MRCI + $Q$ (single and double excitations with Davidson correction) calculations. The calculated potential energy curves (PECs), permanent dipole moment curves (PDMCs), and spectroscopic constants are reported for the 14 lowest electronic states. The comparison of the present results with the rare available theoretical data in literature shows an overall good agreement. To the best of our knowledge, 13 electronic states of the ScSe molecule are not yet investigated either experimentally or theoretically; they are investigated in the present work for the first time.
\end{abstract}

\section{Keywords}

$a b$ initio Calculation, Electronic Structure, Spectroscopic Constants, Potential Energy Curves, Dipole Moments

\section{Introduction}

The electronic structures and nature of chemical bonds between a transition metal and a main group element are of great interest in many areas of science, e.g., in surface chemistry [1], catalysis [2], astrophysics [3]-[5], organometallic chemistry [6], and high-temperature chemistry [7]. They can also serve as simple models for understanding the properties of more complex transition metal compounds. Scandium is the first transition metal atom which has only one d-electron in the ground state. Diatomic molecules like ScSe are simple transition metalcontaining systems in which d-electrons take part in bonding and provide ideal models for understanding the electronic structure and reactivity (chemiluminescent reactions). To the best of our knowledge, no experimental works for the ScSe molecule have been done. The only theoretical works for ScSe in literature are those of Wu

"Corresponding author.

How to cite this paper: Halabi, R. and Korek, M. (2015) Theoretical Calculation of the Low-Lying Electronic States of the Molecule ScSe. Journal of Modern Physics, 6, 2290-2296. http://dx.doi.org/10.4236/jmp.2015.615233 
et al. [8] for the ground $\mathrm{X}^{2} \Sigma^{+}$state using the density functional method.

Because of the lack of studies, either theoretical or experimental, for the excited electronic states of the molecule ScSe and based on our previous studies on diatomic molecules particularly the scandium compounds ScO [9], ScS [10], ScF [11], ScCl [12], ScBr [13] [14] and ScI [15], we present in this work an ab initio investigations of the lowest lying electronic states of the ScSe molecule. In the second part we show that, the calculation has been performed via CAS-SCF/MRCI (Complete Active Space Self Consistent Field, Multireference Configuration Interaction) method. Multireference CI calculations (single and double excitations with Davidson corrections) in which the entire CAS-SCF configuration space was used as the reference, were performed to account the correlation effects. In the third part, the potential energy curves (PECs), the permanent dipole moment curves (DMCs) together with the energy minimum for the ground state $T_{e}$, the equilibrium internuclear distance $R_{e}$, the harmonic frequency $\omega_{e}$ and the rotational constant $B_{e}$ are presented for the 14 lowest-lying electronic states. Thirteen electronic states have been investigated in the present work for the first time.

\section{Method of Calculations}

In the present work, $a b$ initio investigations of the lowest-lying electronic states of the ScSe molecule have been performed via CASSCF method. Multireference MRCI + Q calculations (single and double excitations with Davidson corrections) were performed to determine the correlation effects. The entire CASSCF configuration space was used as a reference in the MRCI calculation which have been performed via the computational chemistry program MOLPRO. The scandium species is treated as a system with 10 inner electrons taken into account using the basis ECP10MDF [16] for s, $\mathrm{p}$ and $\mathrm{d}$ functions for ScSe. The selenium atom is treated as a system with 28 inner electrons taken into account using the basis ECP28MWB [17] including s and p functions. In the range of the internuclear distance $R_{e}$ around equilibrium distances of its ground state, the ScSe molecule is assumed to be mainly ionic as many transition-metals $\mathrm{Sc}^{+} \mathrm{Se}^{-}$. Among the 55 electrons explicitly considered for ScSe (21 electrons for Sc and 34 for Se) 38 inner electrons were frozen in subsequent calculations so that 17 electrons were explicitly treated. The active space contains $6 \sigma$ (Sc: $4 s$; Se: $4 p_{0}, 5 s, 4 d_{0}, 5 p_{0}, 6 s$ ), $3 \pi$ (Se: $4 p_{ \pm 1}$, $4 d_{ \pm 1}, 5 p_{ \pm 1}$ ) and $1 \delta$ (Se: $4 d_{ \pm 2}$ ), this corresponds to 10 active molecular orbitals in the $\mathrm{C}_{2 \mathrm{v}}$ symmetry distributed into irreducible representation in the following way: $7 \mathrm{a}_{1}, 3 \mathrm{~b}_{1}, 3 \mathrm{~b}_{2}, 1 \mathrm{a}_{2}$, noted $[7,3,3,1]$. The doubly occupied orbitals $4 \sigma$ (Sc: $3 s, 3 p_{0}, 3 d_{0}$; Se: $4 s$ ), and $2 \pi$ (Sc: $3 p_{ \pm 1}, 3 d_{ \pm 1}$ ) and $1 \delta$ (Sc: $3 d_{ \pm 2}$ ) have been considered as inactive in the CASSCF calculations. Correlation effects for the seven valence electrons have been taken into account through multireference calculations MRCI + Q (single and double excitations with Davidson correction) where the entire CASSCF configuration space has been used as reference. Calculations have been performed via the computational program MOLPRO ${ }^{1}$ taking advantage of the graphical user interface GABEDIT [18].

\section{Results and Discussion}

The potential energy curves for the doublet and quartet electronic states of ScSe molecule in the representation of ${ }^{2 \mathrm{~s}+1} \Lambda^{(+-)}$have been calculated in term of the internuclear distance in the ranges $2.00 \AA \leq R \leq 3.77 \AA$ (Figures 1-3). The electric dipole moment is a fundamental electrostatic property to study the strength of the long-range dipole-dipole forces, and to understand the macroscopic properties of imperfect gases, liquids and solids. It is demonstrated that the strength of vibrational excitations depends on the variation of the dipole moment as function of geometry such that the DMCs are considered important as they may help to obtain accurate predictions of transition intensities. Hence, the static electric dipole moment of the considered doublet and quartet states are calculated in terms of the internuclear distance $R$ and they will be mainly used here to discuss the bond nature and the polarity and interaction of the states. As the bond nature of an electronic state is related to the magnitude of the dipole moment at a given distance, an ionic nature is indicated from a relatively large magnitude. It is quite common for the molecular electronic states of the potential energy curves to make crossings or avoided crossings. In fact, the avoided crossing regions are likely to be a leakage channels along which the molecules flow from the higher down to the lower potential energy curves. Such crossings or avoided crossings can dramatically alter the stability of the molecules. If these crossings are overlooked, then low barrier transitions can

${ }^{1}$ MOLPRO is a package of $a b$-initio programs written by Werner, H.J., Knowles, P.J., Lindh, R., Manby, F.R., Schütz, M., Celani, P., Korona, T., Rauhut, G., Amos, R.D., Bernhardsson, A., Berning, A., Cooper, D.L., Deegan, M.J.O., Dobbyn, A.J., Eckert, F., Hampel, C., Hetzer, G., Lloyd, A.W., McNicholas, S.J., Meyer, W., Mura, M.E., Nicklass, A., Palmieri, P., Pitzer, R., Schumann, U., Stoll, H., Stone, A.J., Tarroni, R., Thorsteinsson, T. 


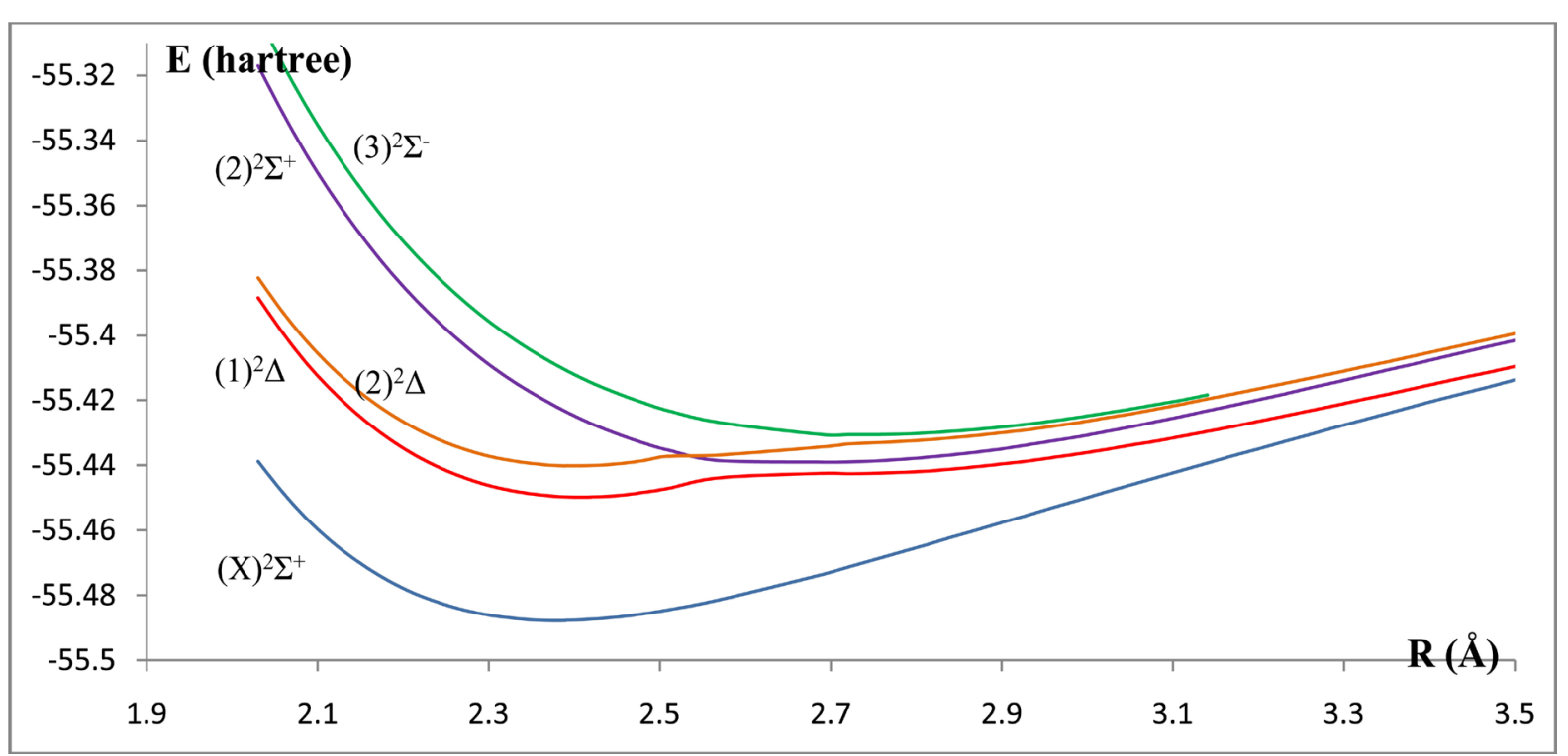

Figure 1. Potential energy curves of the electronic states $\mathrm{X}^{2} \Sigma^{+},(1)^{2} \Delta,(2)^{2} \Delta,(2)^{2} \Sigma^{+}$and $(3)^{2} \Sigma^{-}$of the molecule ScSe.

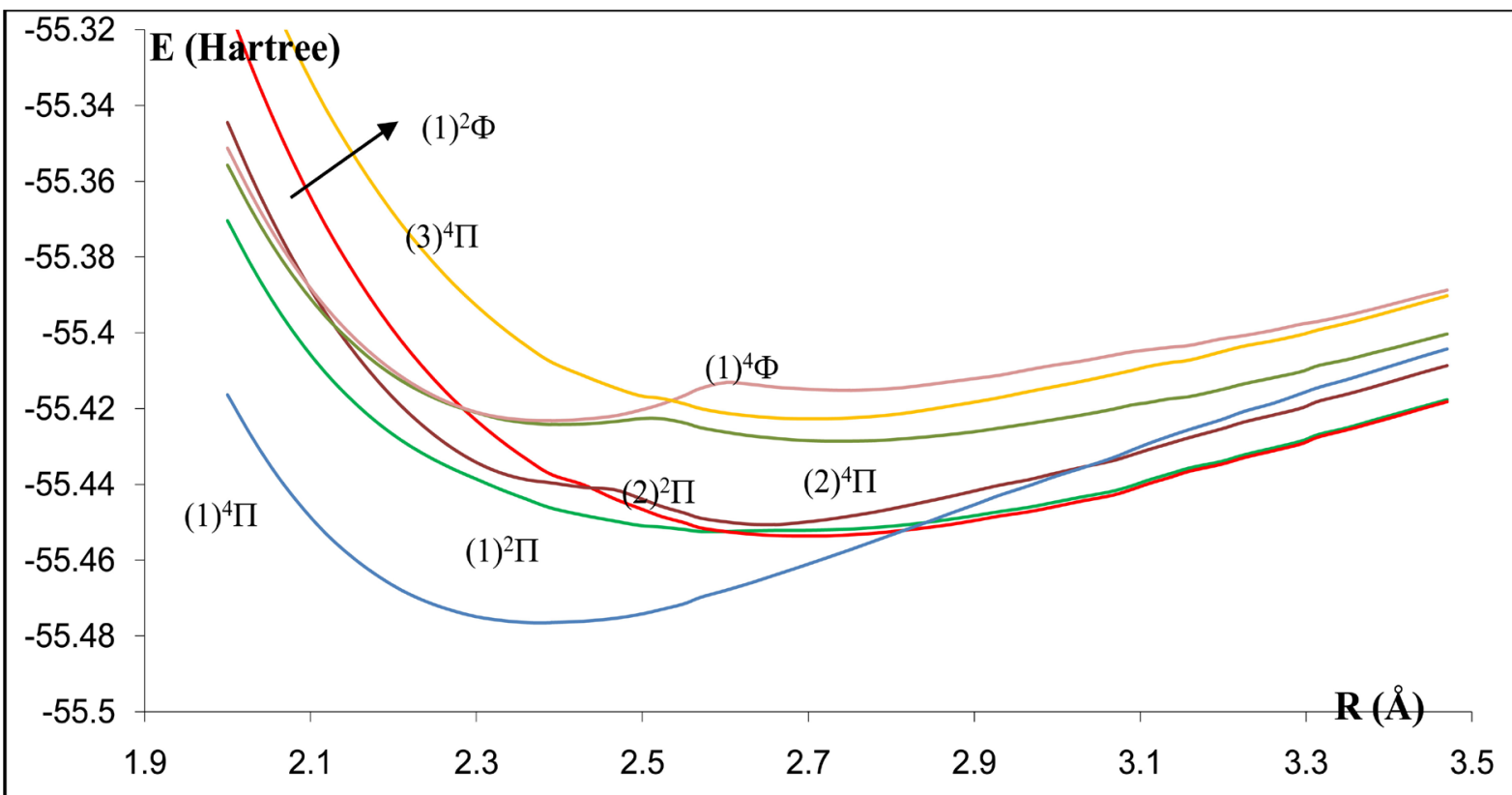

Figure 2. Potential energy curves of the electronic states $(1)^{4} \Pi,(1)^{2} \Pi,(2)^{2} \Pi,(2)^{4} \Pi,(1)^{4} \Phi,(1)^{2} \Phi$ and $(3)^{4} \Pi$ of the molecule ScSe.

be missed and an incorrect chemical picture will arise. In the range of $R$, several avoided crossings have been detected in the potential energy curves for the excited electronic states of the considered molecule ScSe.

For the ScSe molecule, the spectroscopic constants such as the vibration harmonic constant $\omega_{e}$, the internuclear distance at equilibrium $R_{e}$, the rotational constant $B_{e}$ and the electronic transition energy with respect to the ground state $T_{e}$ have been calculated by fitting the energy values around the equilibrium position to a polynomial in terms of the internuclear distance $R$. These values are given in Table 1 together with the available data in the literature. The only values available in literature for the molecule ScSe is that for the ground state $\mathrm{X}^{2} \Sigma^{+}$calculated by using the DFT-B3LYP technique [8]. The comparison of our investigated values of $R_{e}$ for the ground states with those given by Wu et al. [8] for the considered molecule shows a good agreement with the relative differences $\delta R_{e} / R_{e}=4.7 \%$ for the ScSe molecule. A good agreement is obtained by comparing our calculated 


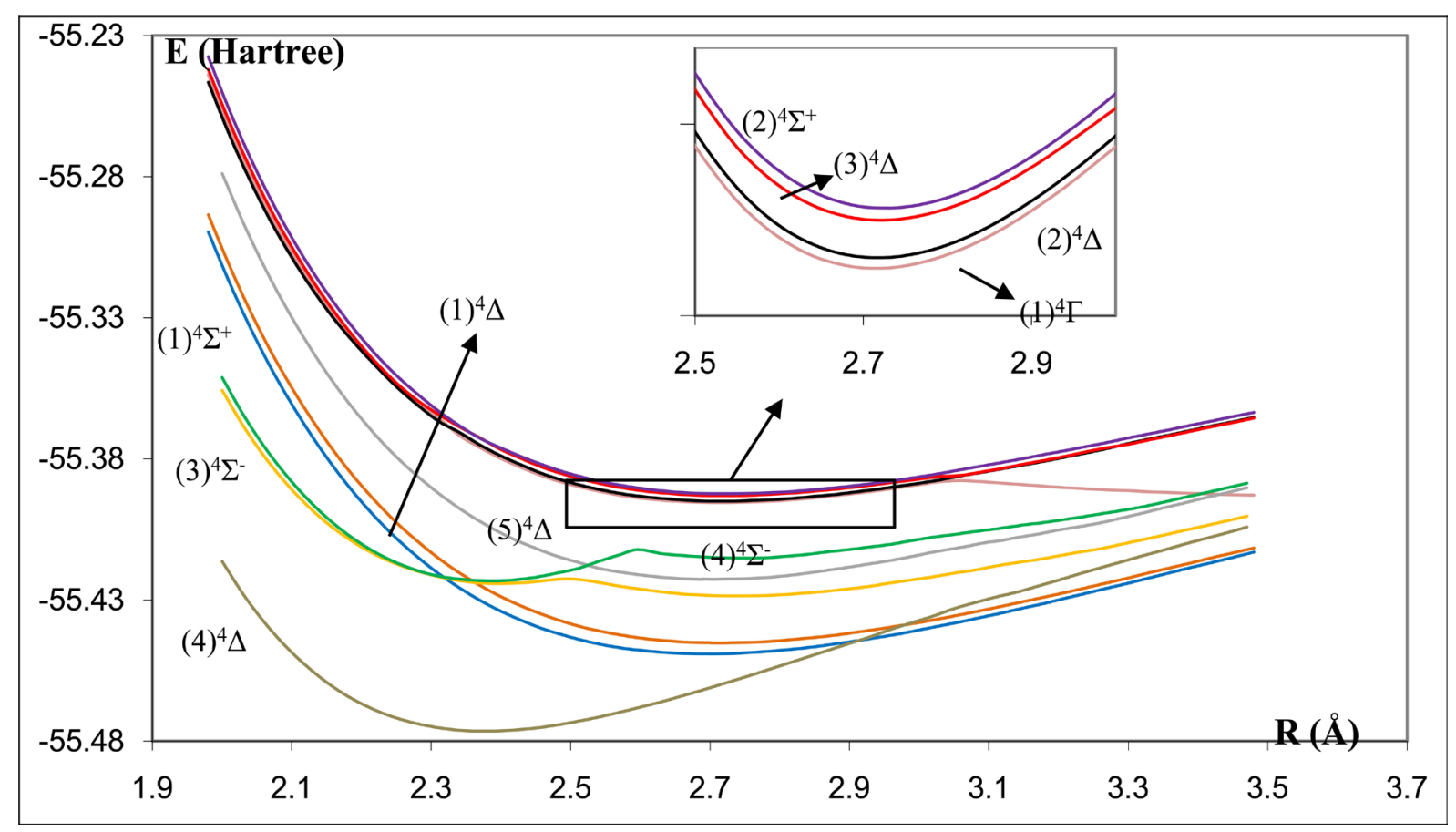

Figure 3. Potential energy curves of the electronic states $(1)^{4} \Sigma^{+},(2)^{4} \Sigma^{+},(3)^{4} \Sigma^{-},(4)^{4} \Sigma^{-},(1)^{4} \Delta,(2)^{4} \Delta,(3)^{4} \Delta,(4)^{4} \Delta$, (5) ${ }^{4} \Delta$, and $(1)^{4} \Gamma$ of the molecule ScSe.

Table 1. Calculated spectroscopic constants for the lowest 14 electronic states of ScSe.

\begin{tabular}{ccccc}
\hline States & $T_{e}\left(\mathrm{~cm}^{-1}\right)$ & $R_{e}(\AA)$ & $\omega_{e}\left(\mathrm{~cm}^{-1}\right)$ & $B_{e} \times 10^{2}\left(\mathrm{~cm}^{-1}\right)$ \\
\hline $\mathrm{X}^{2} \Sigma^{+}$ & $0.0^{\mathrm{a}}$ & $2.3794^{\mathrm{a}}$ & $348.7^{\mathrm{a}}$ & 10.395 \\
$(1)^{2} \Delta$ & $0.0^{\mathrm{b}}$ & $2.272^{\mathrm{b}}$ & $424^{\mathrm{b}}$ & 10.186 \\
$(1)^{4} \Sigma^{+}$ & 8322 & 2.409 & 401.1 & 8.104 \\
$(1)^{4} \Delta$ & 8486 & 2.695 & 252.8 & 7.994 \\
$(3)^{2} \Sigma^{-}$ & 9339 & 2.713 & 246.5 & 7.884 \\
$(2)^{2} \Sigma^{+}$ & 10,269 & 2.732 & 236.6 & 10.213 \\
$(4)^{2} \Sigma^{-}$ & 10,437 & 2.399 & 380.5 & 7.869 \\
$(1)^{4} \Pi$ & 12,540 & 2.735 & 240.5 & 7.878 \\
$(2)^{4} \Pi$ & 12,979 & 2.734 & 252.6 & 9.361 \\
$(2)^{4} \Delta$ & 15,513 & 2.507 & 568.5 & 7.985 \\
$(2)^{4} \Sigma^{+}$ & 20,244 & 2.715 & 234.7 & 7.974 \\
$(1)^{4} \Gamma$ & 20,364 & 2.717 & 237.4 & 7.951 \\
$(3)^{4} \Delta$ & 20,795 & 2.721 & 234.5 & 7.924 \\
$(1)^{4} \Phi$ & 20,933 & 2.725 & 234.3 & 10.417 \\
\hline
\end{tabular}

${ }^{\mathrm{a}}$ Present Theoretical study; ${ }^{\mathrm{b}} \mathrm{Ref}[8]$.

value of $\omega_{e}$ for ScSe molecule with that given in Ref. [8] with the relative difference $\delta \omega_{e} / \omega_{e}=17.6 \%$. No comparisons for the other investigated data for the excited electronic states with other values, since they are given here for the first time.

The dipole moment function of these states exhibits an abrupt change reflecting the avoided crossing between the two states as also observed at the potential energy curves. The positions of these crossings and avoid crossing are given in Table 2 and Table 3.

In Figures 4-6 we show the agreement between the positions of the avoided crossings of the PECs and the 
Table 2. Positions of the avoided crossings between the different electronic states (ScSe).

\begin{tabular}{ccccc}
\hline State 1 & State 2 & Avoided crossing between $\left(\mathrm{n}_{1}\right)$ state $1 /\left(\mathrm{n}_{2}\right)$ state2 & $\mathrm{R}_{\mathrm{AC}}(\AA)$ & $\Delta \mathrm{E}_{\mathrm{AC}}\left(\mathrm{cm}^{-1}\right)$ \\
\hline${ }^{2} \Delta$ & ${ }^{2} \Delta$ & $1 / 2$ & 2.69 & 1779.46 \\
${ }^{2} \Pi$ & ${ }^{2} \Pi$ & $1 / 2$ & 2.60 & 978.78 \\
${ }^{2} \Pi$ & ${ }^{2} \Pi$ & $1 / 2$ & 2.66 & 640.4 \\
${ }^{4} \Pi$ & ${ }^{4} \Pi$ & $2 / 3$ & 2.54 & 1112.04 \\
${ }^{4} \Sigma^{-}$ & ${ }^{4} \Sigma^{-}$ & $3 / 4$ & 2.54 & 1632.84 \\
${ }^{4} \Delta$ & ${ }^{4} \Delta$ & $2 / 3$ & 2.28 & 224.51 \\
${ }^{4} \Delta$ & ${ }^{4} \Delta$ & $2 / 3$ & 3.03 & 534.92 \\
\hline
\end{tabular}

Table 3. Positions of the crossings between the different electronic states (ScSe).

\begin{tabular}{cccc}
\hline State 1 & State 2 & Crossing between $\left(\mathrm{n}_{1}\right)$ state $1 /\left(\mathrm{n}_{2}\right)$ state 2 & $\mathrm{R}_{\mathrm{c}}(\AA)$ \\
\hline${ }^{2} \Sigma$ & ${ }^{2} \Delta$ & $2 / 2$ & 2.57 \\
${ }^{2} \Pi$ & ${ }^{2} \Phi$ & $1 / 1$ & 2.60 \\
${ }^{2} \Pi$ & ${ }^{2} \Phi$ & $2 / 1$ & 2.54 \\
${ }^{4} \Pi$ & ${ }^{2} \Phi$ & $1 / 1$ & 2.81 \\
${ }^{2} \Pi$ & ${ }^{4} \Pi$ & $1 / 1$ & 2.84 \\
${ }^{2} \Pi$ & ${ }^{4} \Pi$ & $2 / 1$ & 3.02 \\
${ }^{2} \Pi$ & ${ }^{4} \Phi$ & $2 / 1$ & 2.09 \\
${ }^{4} \Pi$ & ${ }^{4} \Phi$ & $3 / 1$ & 2.54 \\
${ }^{2} \Pi$ & ${ }^{4} \Pi$ & $2 / 2$ & 2.15 \\
${ }^{4} \Pi$ & ${ }^{2} \Phi$ & $2 / 1$ & 2.30 \\
${ }^{2} \Phi$ & ${ }^{4} \Phi$ & $1 / 1$ & 2.30 \\
${ }^{4} \Sigma^{+}$ & ${ }^{4} \Delta$ & $1 / 4$ & 2.93 \\
${ }^{4} \Sigma^{+}$ & ${ }^{4} \Sigma^{-}$ & $1 / 3$ & 2.33 \\
${ }^{4} \Sigma^{+}$ & ${ }^{4} \Sigma^{-}$ & $1 / 4$ & 2.33 \\
${ }^{4} \Sigma^{-}$ & ${ }^{4} \Delta$ & $3 / 1$ & 2.36 \\
${ }^{4} \Sigma^{-}$ & ${ }^{4} \Delta$ & $4 / 1$ & 2.36 \\
${ }^{4} \Sigma^{-}$ & ${ }^{4} \Delta$ & $4 / 5$ & 2.54 \\
${ }^{4} \Sigma^{-}$ & ${ }^{4} \Delta$ & $4 / 2$ & 3.41 \\
\hline
\end{tabular}

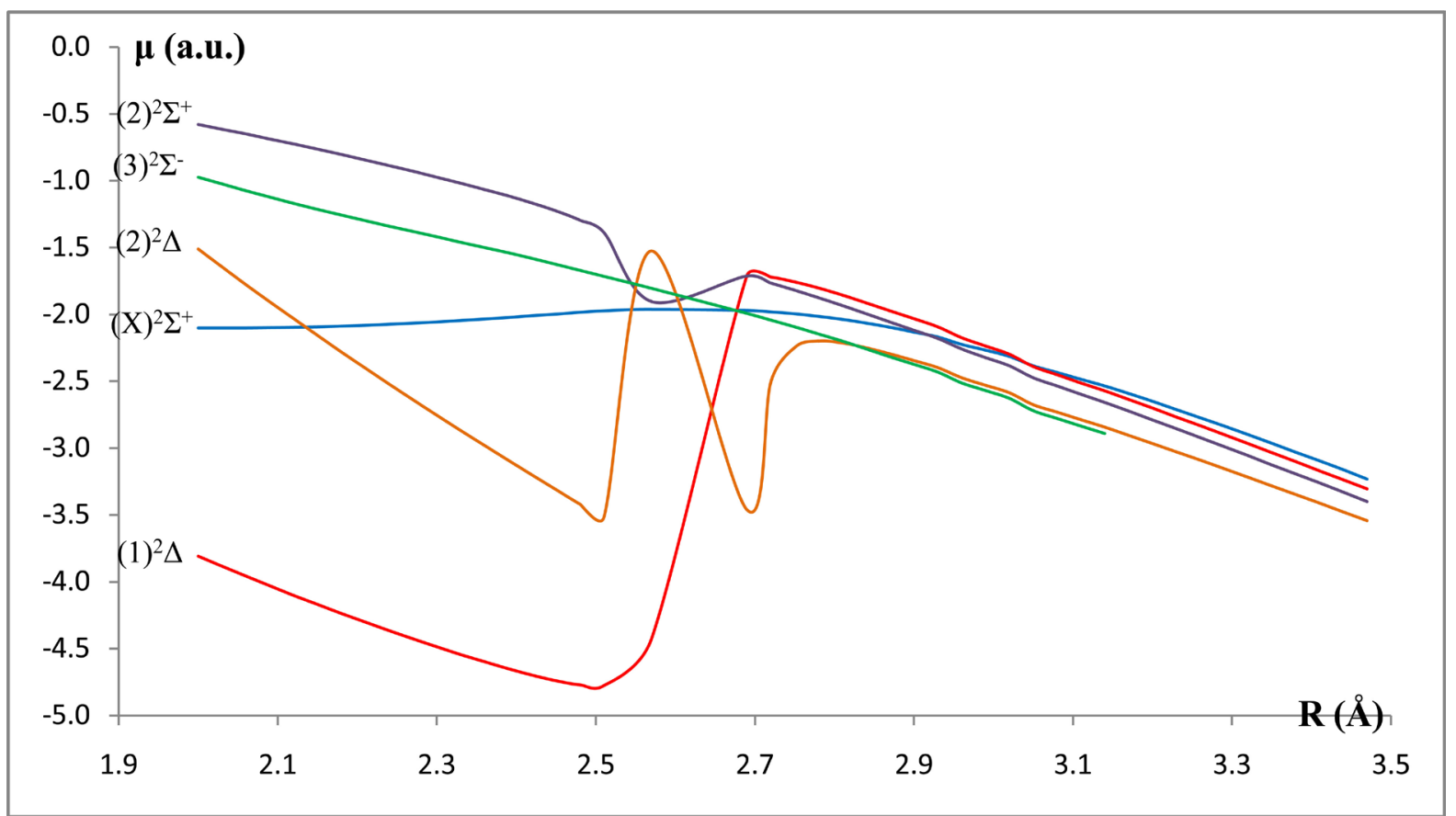

Figure 4. Dipole moment curves of the electronic states $X^{2} \Sigma^{+},(1)^{2} \Delta,(2)^{2} \Delta,(2)^{2} \Sigma^{+}$and $(3)^{2} \Sigma^{-}$of the molecule ScSe. 


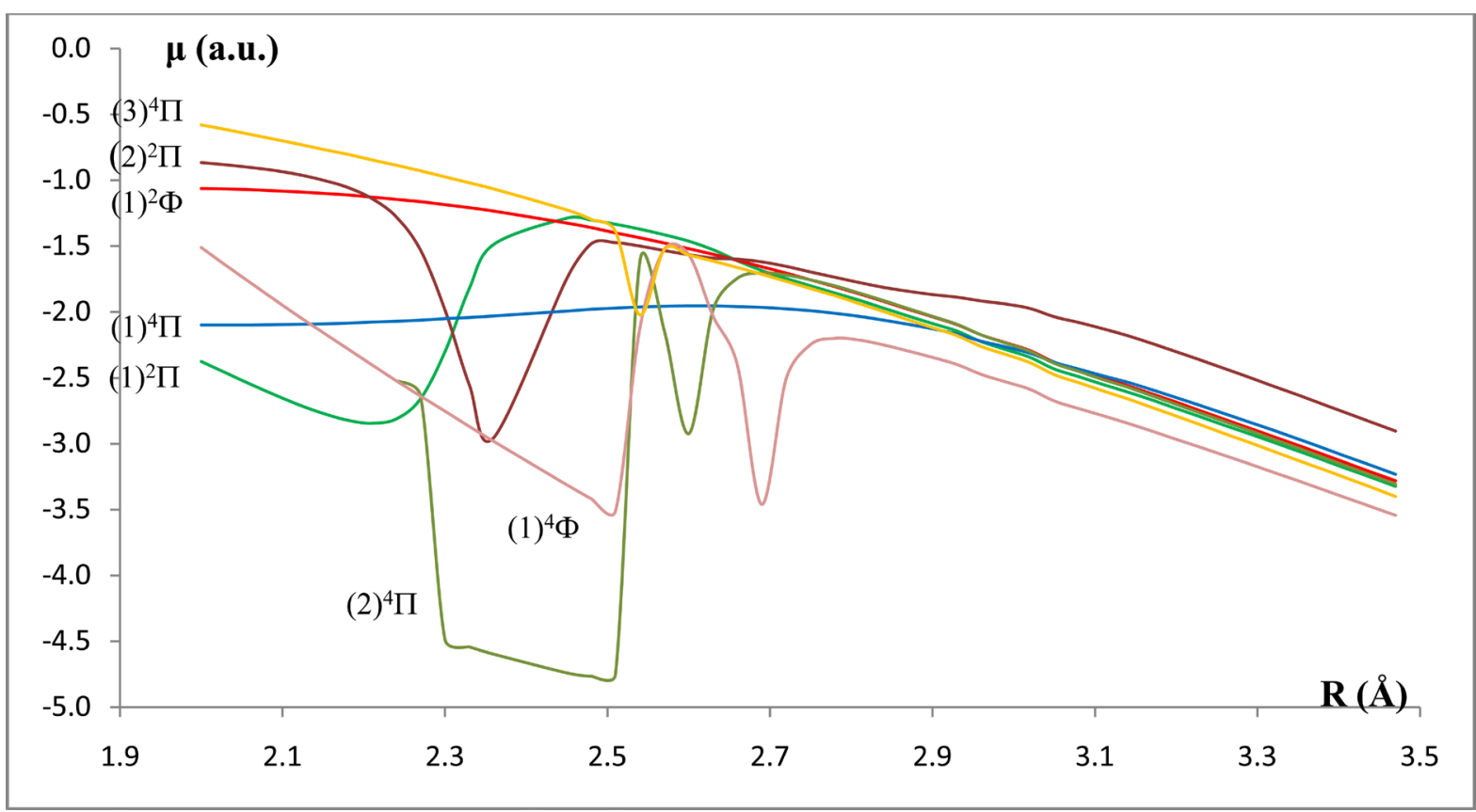

Figure 5. Dipole moment curves of the electronic states $(1)^{4} \Pi,(1)^{2} \Pi,(2)^{2} \Pi,(2)^{4} \Pi,(1)^{4} \Phi,(1)^{2} \Phi$ and (3) $)^{4}$ of the molecule ScSe.

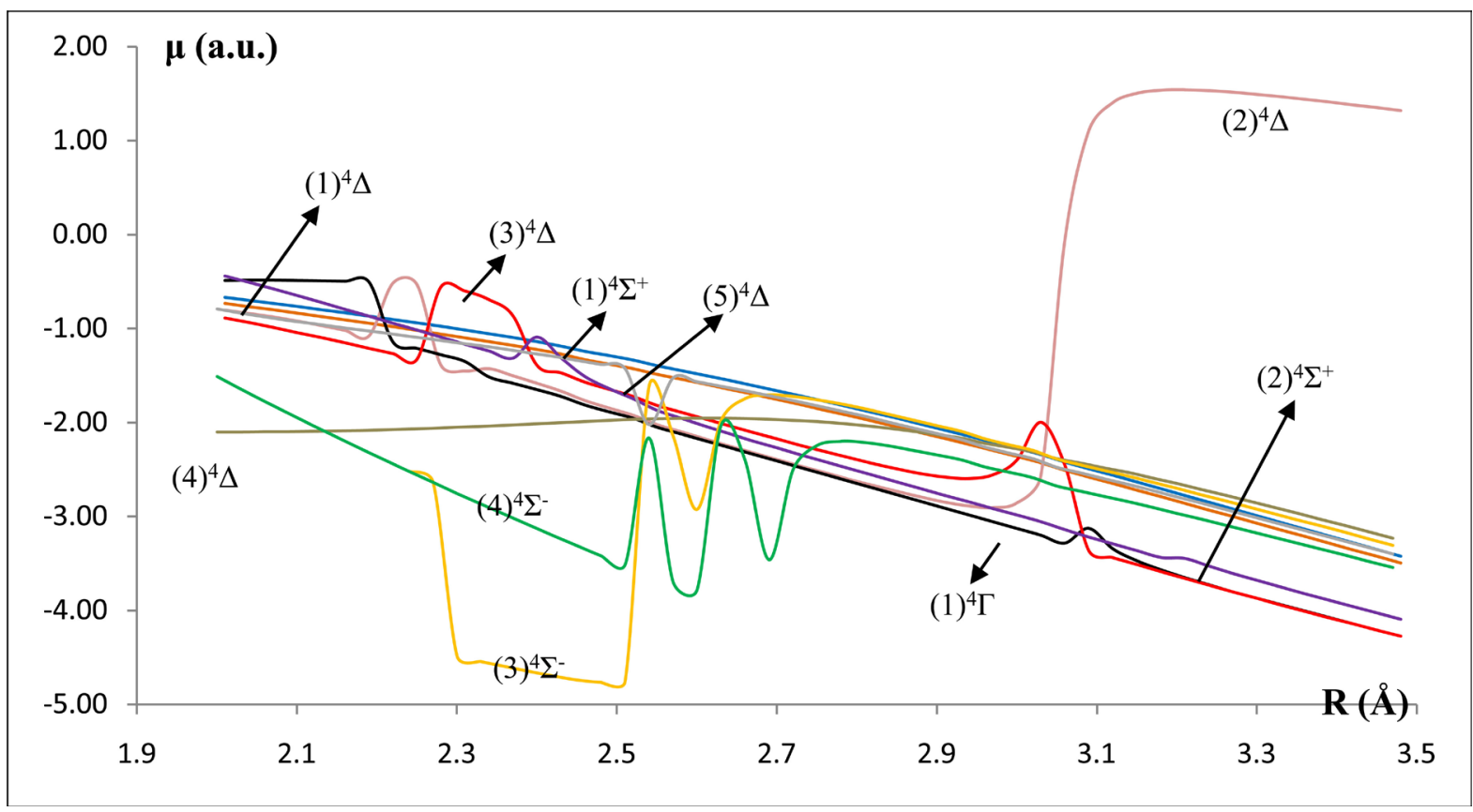

Figure 6. Dipole moment curves of the electronic states $(1)^{4} \Sigma^{+},(2)^{4} \Sigma^{+},(3)^{4} \Sigma^{-}$, (4) $)^{4} \Sigma^{-},(1)^{4} \Delta,(2)^{4} \Delta$, (3) $)^{4} \Delta$, (4) $)^{4} \Delta$, (5) ${ }^{4} \Delta$, and $(1)^{4} \Gamma$ states of the molecule ScSe.

positions of the crossing of the corresponding DMCs. This agreement can be considered as confirmation of the accuracy of the present results on the ScSe molecule. In the present calculation of the DMCs, we considered the Sc atom at the origin. One can notice that, most of the DMCs for the considered molecule are in the negative regions, where the electronegativity of the Sc atom is higher than those of Se atom in these regions and it is independent either on the internuclear distance or the different electronic states. But only for the $(2)^{4} \Delta$ of the ScSe molecule an inversion in the sign is occurred at $R>3 \AA$. At large internuclear distances the decreased value of 
the dipole moment can be explained by the polarization of the orbital in the direction of the negatively charged ligand whereas this orbital polarization tends toward the covalent nature for the electronic state $(2)^{4} \Delta$ of this molecule.

\section{Conclusion}

In the present work, the $a b$ initio investigation for 14 low-lying electronic states of the ScSe molecule has been performed via CASSCF and MRCI + Q (single and double excitations with Davidson correction) calculations. The calculated potential energy curves, the permanent dipole moment curves, and spectroscopic constants $T_{e}, R_{e}$, $\omega_{e}$ and the rotational constant $B_{e}$ are reported for the 14 lowest electronic states of the molecule ScSe. The overall agreement between our calculated values of the spectroscopic constants for the ground states and those of Wu et al. [8] and the agreement between the positions of avoided crossing of the PECs and the crossing of the DMCs for the 13 new excited states confirm the validity and the accuracy of the present results. These investigated data may help for more experimental or theoretical studies in the future for higher electronic states. It is hoped that our computational results will stimulate further experimental studies on such diatomic molecule.

\section{References}

[1] Prutton, M. (1994) Introduction to Surface Physics. 4th Edition, Oxford University Press, New York, 128.

[2] Silberberg, M. (2012) Principles of General Chemistry. 3rd Edition, Science Engineering \& Math, Ch-2 "Molecules, Ions, and Chemical Formulas”.

[3] Maachou, A., Aboura, H., Amrani, B., Khenata, R., Bin Omran, S. and Varshney, D. (2011) Computational Materials Science, 50, 3123-3130. http://dx.doi.org/10.1016/j.commatsci.2011.05.038

[4] Ikrelef, N., Bourbie, D. and Driss-Khodja, K. (2010) Applied Physics Letters, 97, 22101-22103. http://dx.doi.org/10.1063/1.3460289

[5] Maachou, A., Amrani, B. and Driz, M. (2007) Physica B, 388, 384-389. http://dx.doi.org/10.1016/j.physb.2006.06.145

[6] Mackay, R.A. and Henderson, W. (2002) Introduction to Modern Inorganic Chemistry. 6th Edition, CRC Press, Boca Raton.

[7] Bouroushian, M. (2010) Electrochemistry of Metal Chalcogenides. Heidelberg, Berlin, 358. http://dx.doi.org/10.1007/978-3-642-03967-6

[8] Wu, Z.J., Wang, M.Y. and Su, Z.M. (2007) Journal of Computational Chemistry, 28, 703-714. http://dx.doi.org/10.1002/jcc.20603

[9] Srour, J., El-Kork, N. and Korek, M. (2014) Electronic Structure Calculation of the Nanodiatomic Molecule ScO. 14th IEEE International Conference on Nanotechnology, Toronto, 18-21 August 2014, 357-361.

[10] Halabi, R. and Korek, M. (2015) Journal of Modern Physics, 6, 546-552. http://dx.doi.org/10.4236/jmp.2015.65059

[11] Solomonik, V.G. and Mukhanov, A.A. (2012) Journal of Structural Chemistry, 53, 28-34. http://dx.doi.org/10.1134/S0022476612010039

[12] Taher-Mansour, F., Allouche, A.R. and Korek, M. (2009) Journal of Molecular Spectroscopy, 248, 61-65. http://dx.doi.org/10.1016/j.jms.2007.11.012

[13] Korek, K. and Hamdan, A. (2008) International Journal of Quantum Chemistry, 108, 456-461. http://dx.doi.org/10.1002/qua.21524

[14] Hamdan, A. and Korek, M. (2011) Journal of Modern Physics, 2, 1172-1177. http://dx.doi.org/10.4236/jmp.2011.210146

[15] Korek, M., Kontar, S., Taher-Mansour, F. and Allouche, A.R. (2009) International Journal of Quantum Chemistry, 109, 236-242. http://dx.doi.org/10.1002/qua.21779

[16] Jeffrey Hay, P. and Willard Wadt, R. (1985) Journal of Chemical Physics, 82, 270. http://dx.doi.org/10.1063/1.448799

[17] Bergner, A., Dolg, M., Kuechle, W., Stoll, H. and Preuss, H. (1993) Journal of Molecular Physics, 80, $1431-1441$. http://dx.doi.org/10.1080/00268979300103121

[18] Allouche, A.R. (2011) Journal of Computational Chemistry, 32, 174-182. http://dx.doi.org/10.1002/jcc.21600 